\title{
A GLOBAL ESTIMATE FOR THE DIEDERICH-FORNAESS INDEX OF WEAKLY PSEUDOCONVEX DOMAINS
}

\author{
MASANORI ADACHI AND JUDITH BRINKSCHULTE
}

\begin{abstract}
A uniform upper bound for the Diederich-Fornaess index is given for weakly pseudoconvex domains whose Levi form of the boundary vanishes in $\ell$-directions everywhere.
\end{abstract}

\section{$\S 1$. Introduction}

The aim of this article is to reveal a relation between the DiederichFornaess index of weakly pseudoconvex domains and the rank of the Levi form of their boundaries.

Let us first recall the definition of the Diederich-Fornaess index. Consider a complex manifold $X$ and a relatively compact domain $\Omega \Subset X$ with $\mathcal{C}^{2}$ smooth boundary. A defining function of $\Omega$ is a $\mathcal{C}^{2}$-smooth function $\rho: \bar{\Omega} \rightarrow$ $\mathbb{R}$ satisfying $\Omega=\{\rho<0\}$ and whose gradient does not vanish on $\partial \Omega$. To avoid using too many minus signs, we will associate to a fixed defining function $\rho$ the nonnegative function $\hat{\delta}=\hat{\delta}_{\rho}=-\rho$, which can be thought of as a boundary distance function of $\Omega$ with respect to a certain Hermitian metric on $X$ (depending on $\rho$ ).

The Diederich-Fornaess exponent $\eta_{\hat{\delta}}$ of a defining function $-\hat{\delta}$ is the supremum of $\eta \in(0,1)$ such that $-\hat{\delta}^{\eta}$ is a bounded, strictly plurisubharmonic exhaustion function of $\Omega$. If there is no such $\eta$, we let $\eta_{\hat{\delta}}:=0$. The Diederich-Fornaess index $\eta(\Omega)$ of $\Omega$ is the supremum of the DiederichFornaess exponents of defining functions of $\Omega$.

The Diederich-Fornaess index is a numerical index on the strength of a certain pseudoconvexity, more precisely that of hyperconvexity. If $\partial \Omega$ is strictly pseudoconvex, we know that $\partial \Omega$ admits a strictly plurisubharmonic defining function; hence, $\eta(\Omega)=1$. For $\Omega$ to have positive $\eta(\Omega), \Omega$ must be 
Stein, and in fact we need more. A theorem of Ohsawa and Sibony [12, Theorem 1.1]; (see also [11]) tells us that $\eta_{\hat{\delta}}>0$ if and only if $i \partial \bar{\partial}(-\log \hat{\delta}) \geq \omega_{0}$ in $\Omega$ for some Hermitian metric $\omega_{0}$ of $X$. The domains $\Omega$ with positive $\eta(\Omega)$ should carry such a special exhaustion as if they are proper pseudoconvex domains in $X=\mathbb{C P}^{n}$, where Takeuchi's theorem guarantees this kind of exhaustion. Many techniques using such exhaustions have been developed for solving the $\bar{\partial}$-equation on weakly pseudoconvex domains (see, e.g., [2]-[5], [11]).

Let us give several examples to illustrate the situation we are considering. In a celebrated series of works, Diederich and Fornaess (see [7], [8]) showed that, if $X$ is Stein, $\eta(\Omega)>0$ for any domain $\Omega \Subset X$ with $\mathcal{C}^{2}$-smooth pseudoconvex boundary. Note that in this situation $\partial \Omega$ must have a strictly pseudoconvex point, for we can find a level set of a strictly plurisubharmonic exhaustion of $X$ touching $\partial \Omega$ at some points and bounding $\Omega$. They also showed that, for any $\varepsilon>0$, there is $\Omega \Subset X=\mathbb{C}^{2}$ with $0<\eta(\Omega)<\varepsilon$ by using the worm domains, where a Levi-flat portion sits on $\partial \Omega$. Adachi in [1] proved that certain holomorphic disk bundles $\Omega$ over compact Riemann surfaces in their associated flat ruled surfaces $X$ satisfy $\eta(\Omega)>0$ even though $\partial \Omega$ is totally Levi-flat.

A natural question, therefore, is to ask to what extent the DiederichFornaess exponent gets smaller when $\partial \Omega$ is nearly Levi-flat everywhere. Our answer is the following.

Main Theorem. Let $X$ be a complex manifold of dimension $n \geq 2$, and let $\Omega \Subset X$ be a relatively compact domain with $\mathcal{C}^{3}$-smooth boundary. Assume that the Levi form of the boundary $\partial \Omega$ has at least $\ell$ zero eigenvalues everywhere on $\partial \Omega$ where $0 \leq \ell \leq n-1$. Then $\eta(\Omega) \leq(n-\ell) / n$.

In particular, we obtain the following.

Corollary 1.1. If $\eta(\Omega)>1 / n$, then $\partial \Omega$ is not Levi-flat.

Corollary 1.2. If $\eta(\Omega)>(n-1) / n$, then $\partial \Omega$ has a strictly pseudoconvex point.

Let us explain the idea of our proof of the Main Theorem. When $X$ is Stein, we found a strictly pseudoconvex point on $\partial \Omega$ by approximating $\partial \Omega$ by strictly pseudoconvex real hypersurfaces from outside. Since no such approximation exists in general, we use the following method inside. We assume by contradiction that $\eta(\Omega)>(n-\ell) / n$. Then we show in Theorem 4.1, using weighted $L^{2}$-estimates, that any smooth, top-degree form 
with compact support in $\Omega$ is $\bar{\partial}$-exact in the sense of currents on $X$. This is impossible essentially because the top-degree cohomology with compact support does not vanish.

For the proof of Theorem 4.1, we use an estimate of Donnelly-Fefferman type (see [9]) to pass from an $L^{2}$ vanishing result in $L_{n, n}^{2}\left(\Omega, \hat{\delta}^{\eta}\right)$ to an $L^{2}$ vanishing result in $L_{n, n}^{2}\left(\Omega, \hat{\delta}^{-\eta}\right)$. We also modify this argument by using a special Kähler metric $\omega:=i \partial \bar{\partial}\left(-\hat{\delta}^{\eta}\right)$ in $\Omega$ for some $\eta \in\left(0, \eta_{\hat{\delta}}\right)$. This metric respects the degeneracy of the Levi form of $\partial \Omega$ in a certain manner and permits the proof that the trivial extension of this solution is in fact a solution on all of $X$.

\section{$\S 2$. Preliminaries on $L^{2}$-estimate}

In this section we introduce some notation that we use throughout this article. Also, for the convenience of the reader, we recall some of the basic facts concerning a priori estimates and solvability results for the $\bar{\partial}$ operator.

Let $X$ be a complex manifold equipped with a Hermitian metric $\omega_{0}$, and let $\Omega \subset X$ be a domain with $\mathcal{C}^{2}$-smooth boundary. We let $-\hat{\delta}: \bar{\Omega} \rightarrow \mathbb{R}$ be a defining function.

We denote by $L_{p, q}^{2}\left(\Omega, \hat{\delta}^{s}\right)$ the Hilbert space of $(p, q)$-forms $u$ which satisfy

$$
\|u\|_{\hat{\delta}^{s}}^{2}:=\int_{\Omega}|u|_{\omega_{0}}^{2} \hat{\delta}^{s} d V_{\omega_{0}}<+\infty .
$$

Here $d V_{\omega_{0}}$ is the canonical volume element associated with the metric $\omega_{0}$, and $|\cdot|_{\omega_{0}}$ is the norm of $(p, q)$-forms induced by $\omega_{0}$. For $s=0$ the $L^{2}$-spaces just defined coincide with the usual $L^{2}$-spaces on $\Omega$; in this case, we will omit the index $\hat{\delta}^{0}$.

In our proofs it is sometimes necessary to replace the base metric $\omega_{0}$ with a different metric $\omega$. The corresponding Hilbert spaces (resp., norms) will then be denoted by $L_{p, q}^{2}\left(\Omega, \hat{\delta}^{s}, \omega\right)$ (resp., $\left.\|\cdot\|_{\hat{\delta}^{s}, \omega}\right)$.

For later use, we recall the well-known Bochner-Kodaira-Nakano inequality for Kähler metrics for the special case of the trivial line bundle $\mathbb{C}$ on $\Omega$ equipped with a weight function $\varphi \in \mathcal{C}^{2}(\Omega)$, which is the key point when establishing $L^{2}$ existence theorems for the $\bar{\partial}$ operator (see [6]), as follows.

Let $\omega$ be a Kähler metric on $\Omega$. Then for every $u \in \mathcal{D}^{p, q}(\Omega)$ we have

$$
\|\bar{\partial} u\|_{e^{-\varphi}}^{2}+\left\|\bar{\partial}_{e^{-\varphi}}^{*} u\right\|_{e^{-\varphi}}^{2} \geq\langle\langle[i \partial \bar{\partial} \varphi, \Lambda] u, u\rangle\rangle_{e^{-\varphi}} .
$$

Here $\Lambda$ is the adjoint of multiplication by $\omega$. 
A standard computation for the curvature term yields that

$$
\langle[i \partial \bar{\partial} \varphi, \Lambda] u, u\rangle \geq\left(\lambda_{1}+\cdots+\lambda_{q}-\sum_{j=1}^{n} \lambda_{j}\right)|u|^{2}
$$

for any form $u \in \Lambda^{0, q} T^{*} \Omega$. Here $\lambda_{1} \leq \cdots \leq \lambda_{n}$ are the eigenvalues of $i \partial \bar{\partial} \varphi$ with respect to $\omega$.

\section{§3. A special metric}

When $\Omega$ has a defining function $-\hat{\delta}$ with positive Diederich-Fornaess exponent $\eta_{\hat{\delta}}$, taking $0<\eta<\eta_{\hat{\delta}}$, we will equip the domain $\Omega$ with another Kähler metric $\omega:=i \partial \bar{\partial}\left(-\hat{\delta}^{\eta}\right)$ different from $\omega_{0}$.

Let us study the behavior of the metric $\omega$ near $\partial \Omega$ for later use.

LEMMA 3.1. Suppose that $\partial \Omega$ is $\mathcal{C}^{3}$-smooth and that the Levi form of $\partial \Omega$ has at least $\ell$ zero eigenvalues everywhere. Then, we have

$$
d V_{\omega} \lesssim \hat{\delta}^{n \eta-2-(n-\ell-1)} d V_{\omega_{0}}
$$

near $\partial \Omega$.

Proof. First fix a finite covering of $\partial \Omega$ by holomorphic charts $\left\{\left(U ; z_{U}\right)\right\}$ equipped with the Euclidean metrics $\omega_{U}$ associated with their coordinates $z_{U}$. We can fix the covering so that

- $|d \hat{\delta}|_{\omega_{U}}>1$ on each chart $U$;

- $\omega_{U}$ are uniformly comparable to $\omega_{0}$; and

- a $\mathcal{C}^{k}$-norm for functions defined on a neighborhood of $\bar{\Omega}$, say, $\|\cdot\|_{\mathcal{C}^{k}(\bar{\Omega})}$, bounds the $\mathcal{C}^{k}$-norm associated with the coordinate $z_{U}$ from above for functions compactly supported in $U$.

Let $p \in \partial \Omega$, and take one of the holomorphic charts that contains $p$, say, $\left(U ; z_{U}=\left(z_{1}, z_{2}, \ldots, z_{n}\right)\right)$. For small $\varepsilon>0$, consider a nontangential cone $\Gamma_{p, \varepsilon}:=\{z \in U \cap \Omega|| z-p|<2 \hat{\delta}(z)| z-p \mid,<\varepsilon\}$ with vertex at $p$. Note that $\Gamma_{p, \varepsilon}$ is nonempty as $\bar{\Gamma}_{p, \varepsilon}$ contains a segment starting from $p$ normal to ker $d \hat{\delta}_{p}$. It suffices to find a positive constant $C$ independent of the choice of $p$ so that

$$
D_{U}:=\frac{d V_{\omega}}{d V_{\omega_{U}}} \leq C \hat{\delta}^{n \eta-2-(n-\ell-1)}
$$

holds on $\Gamma_{p, \varepsilon}$ for some $\varepsilon=\varepsilon(p)>0$. That is because $\bigcup_{p \in \partial \Omega} \Gamma_{p, \varepsilon(p)}=W \cap \Omega$ for some neighborhood $W$ of $\partial \Omega$ and $\omega_{0}$ is comparable to every $\omega_{U}$ with a uniform constant; we can prove the desired inequality on $W \cap \Omega$. 
To compute $d V_{\omega} / d V_{\omega_{U}}$, we will select an orthonormal frame of $T^{1,0} U$. By a unitary transformation, we can suppose that ker $d \hat{\delta}_{p}=\mathbb{C}^{n-1} \times \mathbb{R}$ and that $\mathbb{C}^{\ell} \times\left\{0^{\prime}\right\}$ is contained in the kernel of the Levi form of $\partial \Omega$ at $p$. Define a $\mathcal{C}^{2}$-smooth frame $\mathcal{Y}=\left(Y_{1}, Y_{2}, \ldots, Y_{n}\right)$ of $T^{1,0} U$ by

$$
Y_{j}:=\frac{\partial}{\partial z_{j}}-\frac{\partial \hat{\delta} / \partial z_{j}}{\partial \hat{\delta} / \partial z_{n}} \frac{\partial}{\partial z_{n}} \quad(j=1,2, \ldots, n-1), \quad Y_{n}:=\frac{\partial}{\partial z_{n}}
$$

Note that $\left\{Y_{1}, Y_{2}, \ldots, Y_{n-1}\right\}$ spans ker $\partial \hat{\delta}$ on $U$. We apply the Gram-Schmidt procedure to $\mathcal{Y}$ and obtain an orthonormal frame $\mathcal{X}=\left(X_{1}, X_{2}, \ldots, X_{n}\right)$ with respect to $\omega_{U}$. Denote by $A(z)=\left(a_{j k}(z)\right)$ the change-of-base matrices at each point: $X_{k}=\sum_{j=1}^{n} Y_{j} a_{j k}$ on $U$.

We would like to estimate each $\lambda_{j \bar{k}}:=\omega\left(X_{j}, \overline{X_{k}}\right)$ on $\Gamma_{p, \varepsilon}$. To achieve it, we combine two estimates: one is about $\mu_{j \bar{k}}:=\omega\left(Y_{j}, \overline{Y_{k}}\right)$, and the other is about the change-of-base matrices $A(z)$.

First consider the behavior of $\mu_{j \bar{k}}$ on $\Gamma_{p, \varepsilon}$. The equality

$$
\omega=i \eta \hat{\delta}^{\eta}\left\{\frac{\partial \bar{\partial}(-\hat{\delta})}{\hat{\delta}}+(1-\eta) \frac{\partial \hat{\delta} \wedge \bar{\partial} \hat{\delta}}{\hat{\delta}^{2}}\right\}
$$

yields that, if $j=k=n$,

$$
\lim _{z \rightarrow p, z \in U \cap \Omega} \frac{\mu_{n \bar{n}}(z)}{\hat{\delta}(z)^{\eta-2}}=\eta(1-\eta)\left|\partial \hat{\delta}\left(Y_{n}(p)\right)\right|^{2} \leq\|\hat{\delta}\|_{\mathcal{C}^{1}(\bar{\Omega})}^{2} ;
$$

otherwise,

$$
\lim _{z \rightarrow p, z \in U \cap \Omega} \frac{\left|\mu_{j \bar{k}}(z)\right|}{\hat{\delta}(z)^{\eta-1}}=\eta\left|\partial \bar{\partial}(-\hat{\delta})\left(Y_{j}(p), \overline{Y_{k}(p)}\right)\right| \leq\|\hat{\delta}\|_{\mathcal{C}^{2}(\bar{\Omega})}
$$

We can say more for directions in which the Levi form vanishes. If $1 \leq j \leq \ell$, $1 \leq k \leq n-1$ or $1 \leq j \leq n-1,1 \leq k \leq \ell$,

$$
\begin{aligned}
& \limsup _{z \rightarrow p, z \in \Gamma_{p, \varepsilon}} \frac{\left|\mu_{j \bar{k}}(z)\right|}{\hat{\delta}(z)^{\eta}} \\
& =\limsup _{z \rightarrow p, z \in \Gamma_{p, \varepsilon}} \eta\left|\frac{\partial \bar{\partial}(-\hat{\delta})\left(Y_{j}(z), \overline{Y_{k}(z)}\right)}{\hat{\delta}(z)}\right| \\
& =\limsup _{z \rightarrow p, z \in \Gamma_{p, \varepsilon}} \eta \frac{|z-p|}{\hat{\delta}(z)}\left|\frac{\partial \bar{\partial}(-\hat{\delta})\left(Y_{j}(z), \overline{Y_{k}(z)}\right)-0}{|z-p|}\right|
\end{aligned}
$$




$$
\begin{aligned}
& \leq 2\left|d\left(\partial \bar{\partial}(-\hat{\delta})\left(Y_{j}, \overline{Y_{k}}\right)\right)(p)\right|_{\omega_{U}} \\
& \leq 2\left(\|\hat{\delta}\|_{\mathcal{C}^{3}(\bar{\Omega})}+2\|\hat{\delta}\|_{\mathcal{C}^{2}(\bar{\Omega})}^{2}\right) .
\end{aligned}
$$

Next we proceed to estimate the change-of-base matrices $A(z)$. We identify an $n$-tuple of $(1,0)$-vectors with an $n \times n$ matrix by using our coordinate $z_{U}$. Then, we have $\mathcal{X}(p)=\mathcal{Y}(p)=I_{n}$ and $A(z)=\mathcal{Y}^{-1}(z) \cdot \mathcal{X}(z)$, where $I_{n}$ denotes the identity matrix. As a matrix-valued 1-form, we have

$$
d A(p)=\mathcal{Y}^{-1}(p) \cdot d \mathcal{X}(p)+d \mathcal{Y}^{-1}(p) \cdot X(p)=d \mathcal{X}(p)+d \mathcal{Y}^{-1}(p)
$$

Since $I_{n}=\mathcal{Y}^{-1}(z) \cdot \mathcal{Y}(z)$, we also have

$$
0=d\left(\mathcal{Y}^{-1} \cdot \mathcal{Y}\right)(p)=d \mathcal{Y}^{-1}(p)+d \mathcal{Y}(p)
$$

Now let GS : GL $(n, \mathbb{C}) \rightarrow U(n)$ be the map determined by the Gram-Schmidt procedure. Its differential at $I_{n}$ defines $d \mathrm{GS}_{I_{n}}: \mathfrak{g l}(n, \mathbb{C}) \rightarrow \mathfrak{u}(n)$. We linearly extend this map on matrix-valued, that is, $\mathfrak{g l}(n, \mathbb{C})$-valued 1 -forms, and we also write $d \mathrm{GS}_{I_{n}}$ for the extended linear map by abuse of notation. Then, $d \mathrm{GS}_{I_{n}}(d \mathcal{Y}(p))=d \mathcal{X}(p)$ follows from $\operatorname{GS}(\mathcal{Y}(z))=\mathcal{X}(z)$. Combining these equalities, we therefore have

$$
d A(p)=d \mathrm{GS}_{I_{n}}(d \mathcal{Y}(p))-d \mathcal{Y}(p)
$$

We use the norm $|A|=\max _{j, k}\left|a_{j k}\right|$ for matrices, and we consider the induced norm for linear maps between spaces of matrices. Since a straightforward computation yields $|d \mathcal{Y}(p)|_{\omega_{U}} \leq\|\hat{\delta}\|_{\mathcal{C}^{2}(\bar{\Omega})}$, we have

$$
\begin{aligned}
\limsup _{z \rightarrow p, z \in \Gamma_{p, \varepsilon}} \frac{\left|A(z)-I_{n}\right|}{\hat{\delta}(z)} & =\limsup _{z \rightarrow p, z \in \Gamma_{p, \varepsilon}} \frac{|z-p|}{\hat{\delta}(z)} \frac{\left|A(z)-I_{n}\right|}{|z-p|} \\
& \leq 2|d A(p)|_{\omega_{U}} \\
& \leq 2\left(\left|d \mathrm{GS}_{I_{n}}\right|+1\right)|d \mathcal{Y}(p)|_{\omega_{U}} \\
& \leq 2\left(\left|d \mathrm{GS}_{I_{n}}\right|+1\right)\|\hat{\delta}\|_{\mathcal{C}^{2}(\bar{\Omega})} .
\end{aligned}
$$

Note that $\left|d \mathrm{GS}_{I_{n}}\right|$ is independent of $p$ and depends only on $n$. 
By combining the estimates on $\mu_{j \bar{k}}$ and $A(z)$ above, we can find a positive constant $C$ depending only on $n=\operatorname{dim} X$ and $\|\hat{\delta}\|_{\mathcal{C}^{3}(\bar{\Omega})}$ so that

$$
\begin{aligned}
\left|\lambda_{j \bar{k}}(z)\right| & =\left|\sum_{l, m} \mu_{l \bar{m}}(z) a_{j l}(z) \overline{a_{k m}(z)}\right| \\
\leq & \begin{cases}C \hat{\delta}^{\eta-2} & (\text { for } j=k=n) \\
C \hat{\delta}^{\eta} & (\text { for } 1 \leq j \leq \ell, 1 \leq k \leq n-1) \\
C \hat{\delta}^{\eta} & \text { (for } 1 \leq j \leq n-1,1 \leq k \leq \ell) \\
C \hat{\delta}^{\eta-1} & \text { (otherwise) }\end{cases}
\end{aligned}
$$

holds on $\Gamma_{p, \varepsilon}$ for $0<\varepsilon \ll 1$. It follows that

$$
\begin{aligned}
D_{U} & =\operatorname{det}\left(\lambda_{j \bar{k}}\right)_{j, k=1}^{n} \\
& \leq n ! C^{n} \hat{\delta}^{\ell \eta+(n-\ell-1)(\eta-1)+(\eta-2)} \\
& =n ! C^{n} \hat{\delta}^{n \eta-2-(n-\ell-1)}
\end{aligned}
$$

on $\Gamma_{p, \varepsilon}$, which completes the proof.

Lemma 3.2. Suppose that $\partial \Omega$ is $\mathcal{C}^{3}$-smooth and that the Levi form of $\partial \Omega$ has at least $\ell$ zero eigenvalues everywhere. Then, for any $(n, n-1)$-form $u$ on $\Omega$,

$$
|u|_{\omega_{0}}^{2} d V_{\omega_{0}} \lesssim|u|_{\omega}^{2} \hat{\delta}^{(n-1) \eta-2-(n-\ell-1)} d V_{\omega}
$$

near $\partial \Omega$ with positive constant independent of $u$.

Proof. It suffices to prove the inequality on $\Gamma_{p, \varepsilon}$ with $\omega_{U}$ instead of $\omega_{0}$, where we work in the same local situation as in the proof of Lemma 3.1. Consider the induced frame of $\wedge^{n} T^{1,0} U \otimes \wedge^{n-1} T^{0,1} U$ from $\left\{X_{1}, X_{2}, \ldots, X_{n}\right\}$ over $U$. It follows from (3.3) that

$$
\begin{aligned}
& \left|X_{1} \wedge X_{2} \wedge \cdots \wedge X_{n} \otimes \bar{X}_{1} \wedge \bar{X}_{2} \wedge \cdots \wedge \widehat{\bar{X}}_{k} \wedge \cdots \wedge \bar{X}_{n}\right|_{\omega}^{2} \\
& \quad=D_{U}\left|\bar{X}_{1} \wedge \bar{X}_{2} \wedge \cdots \wedge \widehat{\bar{X}}_{k} \wedge \cdots \wedge \bar{X}_{n}\right|_{\omega}^{2} \\
& \quad \leq D_{U}(n-1) ! C^{n-1} \begin{cases}\hat{\delta}^{(\ell-1) \eta+(n-\ell-1)(\eta-1)+(\eta-2)} & (\text { for } 1 \leq k \leq \ell) \\
\hat{\delta}^{\ell \eta+(n-\ell-2)(\eta-1)+(\eta-2)} & (\text { for } \ell+1 \leq k \leq n-1) \\
\hat{\delta}^{\ell \eta+(n-\ell-1)(\eta-1)} & (\text { for } k=n)\end{cases} \\
& \quad \leq D_{U}(n-1) ! C^{n-1} \hat{\delta}^{(n-1) \eta-2-(n-\ell-1)} .
\end{aligned}
$$


Hence, we can estimate $|u|_{\omega}^{2}$ as

$$
\begin{aligned}
|u|_{\omega}^{2} & \geq \max _{1 \leq k \leq n} \frac{\left|u\left(X_{1}, X_{2}, \ldots, X_{n}, \bar{X}_{1}, \bar{X}_{2}, \ldots, \widehat{\bar{X}}_{k}, \ldots, \bar{X}_{n}\right)\right|^{2}}{\left|X_{1} \wedge X_{2} \wedge \cdots \wedge X_{n} \otimes \bar{X}_{1} \wedge \bar{X}_{2} \wedge \cdots \wedge \widehat{\bar{X}}_{k} \wedge \cdots \wedge \bar{X}_{n}\right|_{\omega}^{2}} \\
& \geq \frac{\max _{1 \leq k \leq n}\left|u\left(X_{1}, X_{2}, \ldots, X_{n}, \bar{X}_{1}, \bar{X}_{2}, \ldots, \widehat{\bar{X}}_{k}, \ldots, \bar{X}_{n}\right)\right|^{2}}{(n-1) ! C^{n-1} D_{U} \hat{\delta}^{(n-1) \eta-2-(n-\ell-1)}} \\
& \geq C^{\prime} \frac{|u|_{\omega_{U}}^{2}}{D_{U} \hat{\delta}^{(n-1) \eta-2-(n-\ell-1)}},
\end{aligned}
$$

with constant $C^{\prime}>0$ independent of $u$. We therefore have the desired inequality

$$
\begin{aligned}
|u|_{\omega}^{2} d V_{\omega} & \geq C^{\prime} \frac{1}{D_{U}}|u|_{\omega_{U}}^{2} \hat{\delta}^{-(n-1) \eta+2+(n-\ell-1)} D_{U} d V_{\omega_{U}} \\
& =C^{\prime}|u|_{\omega_{U}}^{2} \hat{\delta}^{-(n-1) \eta+2+(n-\ell-1)} d V_{\omega_{U}}
\end{aligned}
$$

\section{$\S 4$. The $\bar{\partial}$ equation in top degree}

In this section we will study a version of an $L^{2} \bar{\partial}$-Cauchy problem in top degree on a smoothly bounded domain with weakly pseudoconvex boundary, which, by duality, implies a restriction on the rank of the Levi form of $\partial \Omega$.

THEOREM 4.1. Let $X$ be a complex manifold of dimension $n \geq 2$, and let $\Omega \Subset X$ be a relatively compact domain with $\mathcal{C}^{3}$-smooth boundary. Suppose that the Levi form of $\partial \Omega$ has at least $\ell$ zero eigenvalues everywhere on $\partial \Omega$ for some $0 \leq \ell \leq n-1$. If $\eta(\Omega)>(n-\ell) / n$, then for any $f \in L_{n, n}^{2}(X)$ which is compactly supported in $\Omega$ there exists a current $T \in \mathcal{D}_{0,1}^{\prime}(X)$ supported in $\bar{\Omega}$ such that $\bar{\partial} T=f$ in the distribution sense on $X$.

Theorem 4.1 is based on the following estimate of Donnelly-Fefferman type.

THEOREM 4.2. Let $X$ be a complex manifold of dimension $n \geq 2$, and let $\Omega \Subset X$ be a relatively compact domain with $\mathcal{C}^{2}$-smooth boundary. Let $-\hat{\delta}$ be a defining function of $\Omega$ with Diederich-Fornaess exponent $\eta_{\hat{\delta}}>0$. For an arbitrary but fixed $\eta \in\left(0, \eta_{\hat{\delta}}\right)$ we define $\omega:=i \partial \bar{\partial}\left(-\hat{\delta}^{\eta}\right)$. Then, for any $f \in L_{n, n}^{2}\left(\Omega, \hat{\delta}^{-\eta}, \omega\right)$, there exists $u \in L_{n, n-1}^{2}\left(\Omega, \hat{\delta}^{-\eta}, \omega\right)$ satisfying $\bar{\partial} u=f$ in the distribution sense in $\Omega$. 
Proof. Let us first see that the conclusion follows in a standard manner from the following a priori estimate.

Claim. There exists a constant $C>0$ such that

$$
\|v\|_{\hat{\delta}^{-\eta}, \omega}^{2} \leq C\left\|\bar{\partial}^{*} v\right\|_{\hat{\delta}^{-\eta}, \omega}^{2}
$$

for any $v \in \mathcal{D}^{n, n}(\Omega)$. Here $\bar{\partial}^{*}=\bar{\partial}_{\hat{\delta}^{-\eta}, \omega}^{*}$ is the adjoint of $\bar{\partial}$ with respect to the scalar product induced by $\|\cdot\|_{\hat{\delta}^{-\eta}, \omega}$.

Note that in the top degree we can work with noncomplete metrics, since there is no compatibility condition. Indeed, let us take $f \in L_{n, n}^{2}\left(\Omega, \hat{\delta}^{-\eta}, \omega\right)$ and define a linear functional $\phi$ on $\bar{\partial}^{*}\left(\mathcal{D}^{n, n}(\Omega)\right) \subset L_{n, n-1}^{2}\left(\Omega, \hat{\delta}^{-\eta}, \omega\right)$ by $\phi\left(\bar{\partial}^{*} v\right)=\langle\langle v, f\rangle\rangle_{\hat{\delta}^{-\eta}, \omega}$, which is well defined and bounded from (4.1). The Hahn-Banach theorem allows us to extend $\phi$ to a bounded linear functional on $L_{n, n-1}^{2}\left(\Omega, \hat{\delta}^{-\eta}, \omega\right)$, and the Riesz representation theorem yields $u \in L_{n, n-1}^{2}\left(\Omega, \hat{\delta}^{-\eta}, \omega\right)$ satisfying

$$
\left\langle\left\langle\bar{\partial}^{*} v, u\right\rangle\right\rangle_{\hat{\delta}^{-\eta}, \omega}=\langle\langle v, f\rangle\rangle_{\hat{\delta}^{-\eta}, \omega}
$$

for all $v \in \mathcal{D}^{n, n}(\Omega)$; that is, $\bar{\partial} u=f$ in the distribution sense in $\Omega$.

Let us proceed to prove (4.1). For a direct proof of it, we would have to work with different adjoint operators. Therefore, it is somewhat more convenient to actually prove the dual a priori estimate

$$
\|v\|_{\hat{\delta}^{\eta}, \omega} \leq C\|\bar{\partial} v\|_{\hat{\delta}^{\eta}, \omega}
$$

for any $v \in \mathcal{D}^{0,0}(\Omega)$. Equation (4.1) then follows from (4.2) using a weighted Hodge star operator.

So let us proceed to prove (4.2). Since $\eta<\eta_{\hat{\delta}}$, there exists some small $\varepsilon>0$ such that $\eta+\varepsilon<\eta_{\hat{\delta}}$, which means that

$$
i \partial \bar{\partial}\left(-\hat{\delta}^{\eta+\varepsilon}\right) \geq 0 \quad \text { in } \Omega .
$$

But then

$i \partial \bar{\partial} \log \hat{\delta}^{\eta+\varepsilon}=\frac{i \partial \bar{\partial} \hat{\delta}^{\eta+\varepsilon}}{\hat{\delta}^{\eta+\varepsilon}}-i \partial \log \hat{\delta}^{\eta+\varepsilon} \wedge \bar{\partial} \log \hat{\delta}^{\eta+\varepsilon} \leq-i \partial \log \hat{\delta}^{\eta+\varepsilon} \wedge \bar{\partial} \log \hat{\delta}^{\eta+\varepsilon}$

Hence, we get

$$
\operatorname{Trace}_{\omega}\left(i \partial \bar{\partial} \log \hat{\delta}^{\eta+\varepsilon}\right) \leq-\left|\bar{\partial} \log \hat{\delta}^{\eta+\varepsilon}\right|_{\omega}^{2} \quad \text { in } \Omega
$$


Putting $\psi=\hat{\delta}^{\eta}$, we have $i \partial \bar{\partial} \psi=-\omega$ by definition of $\omega$; thus, Trace $\omega(i \partial \bar{\partial} \psi)=$ $-n$. Hence, we get

$$
\operatorname{Trace}_{\omega}\left(i \partial \bar{\partial} \psi+i \partial \bar{\partial} \log \hat{\delta}^{\eta+\varepsilon}\right) \leq-n-\left|\partial \log \hat{\delta}^{\eta+\varepsilon}\right|_{\omega}^{2} \quad \text { on } \Omega
$$

On $\Omega$, we consider the weight function $e^{-\psi}$. Since $e^{-\psi}$ is bounded from below and from above by positive constants on $\Omega$, we can replace the norm $\|\cdot\|$ by $\|\cdot\|_{e^{-\psi}}$ for forms on $\Omega$.

Multiplying the metric of the trivial bundle $\mathbb{C}$ further by $\hat{\delta}^{-(\eta+\varepsilon)}=$ $e^{-\log \hat{\delta}^{\eta+\varepsilon}}$ on $\Omega$, it then follows from (2.1) and (2.2) that for $u \in \mathcal{D}^{0,0}(\Omega)$ one has

$$
\left\langle\left\langle-\operatorname{Trace}_{\omega}\left(i \partial \bar{\partial} \psi+i \partial \bar{\partial} \log \hat{\delta}^{\eta+\varepsilon}\right) u, u\right\rangle\right\rangle_{e^{-\psi} \hat{\delta}^{-(\eta+\varepsilon)}, \omega} \leq\|\bar{\partial} u\|_{e^{-\psi} \hat{\delta}^{-(\eta+\varepsilon)}, \omega}^{2}
$$

Using (4.3) we obtain

$$
\left\langle\left\langle\left(n+\left|\bar{\partial} \log \hat{\delta}^{\eta+\varepsilon}\right|_{\omega}^{2}\right) u, u\right\rangle\right\rangle_{e^{-\psi} \hat{\delta}^{-(\eta+\varepsilon)}, \omega} \leq\|\bar{\partial} u\|_{e^{-\psi} \hat{\delta}^{-(\eta+\varepsilon)}, \omega}^{2}
$$

for $u \in \mathcal{D}^{0,0}(\Omega)$. Observing that $\partial \log \hat{\delta}^{\eta+\varepsilon}=(\eta+\varepsilon) \partial \log \hat{\delta}$ and setting $u=$ $v \hat{\delta}^{\eta+\varepsilon / 2}$, we obtain

$$
\begin{aligned}
& \left\langle\left\langle\left(n+(\eta+\varepsilon)^{2}|\bar{\partial} \log \hat{\delta}|_{\omega}^{2}\right) v, v\right\rangle\right\rangle_{e^{-\psi} \hat{\delta}^{\eta}, \omega} \\
& \leq\left\|\bar{\partial} v+\left(\eta+\frac{\varepsilon}{2}\right) v \bar{\partial} \log \hat{\delta}\right\|_{e^{-\psi} \hat{\delta}^{\eta}, \omega}^{2} \\
& \leq\left(1+\frac{1}{a}\right)\|\bar{\partial} v\|_{e^{-\psi} \hat{\delta}^{\eta}, \omega}^{2}+(1+a)\left(\eta+\frac{\varepsilon}{2}\right)^{2}\|v \bar{\partial} \log \hat{\delta}\|_{e^{-\psi} \hat{\delta}^{\eta}, \omega}^{2}
\end{aligned}
$$

Choosing $a$ so small that $(1+a)(\eta+\varepsilon / 2)^{2} \leq(\eta+\varepsilon)^{2}$, we can thus absorb the last term in (4.4) in the left-hand side, which immediately gives the a priori estimate (4.2).

Now let us give the proof of Theorem 4.1.

Proof of Theorem 4.1. By the assumption on $\Omega$, we can find a defining function $-\hat{\delta}$ with $\eta_{\hat{\delta}}>(n-\ell) / n$. We fix some real $\eta$ such that $(n-\ell) / n<$ $\eta<\eta_{\hat{\delta}}$, and we apply Theorem 4.2 with this choice of $\eta$. 
Now let $f \in L_{n, n}^{2}(X)$ be compactly supported in $\Omega$, which implies that $f \in L_{n, n}^{2}\left(\Omega, \hat{\delta}^{-\eta}, \omega\right)$. Hence, it follows from Theorem 4.2 that there exists $u \in L_{n, n-1}^{2}\left(\Omega, \hat{\delta}^{-\eta}, \omega\right)$ satisfying $\bar{\partial} u=f$ in $\Omega$.

We first claim that, if we extend $u$ by zero outside $\Omega$, then it defines a current $T=T_{u} \in \mathcal{D}_{0,1}^{\prime}(X)$. Indeed, we see from Lemma 3.2 that

$$
\int_{\Omega}|u|_{\omega_{0}}^{2} \hat{\delta}^{1-\nu} d V_{\omega_{0}} \lesssim \int_{\Omega}|u|_{\omega}^{2} \hat{\delta}^{1-\nu} \hat{\delta}^{(n-1) \eta-2-(n-\ell-1)} d V_{\omega} .
$$

Now a straightforward computation shows that the last integral can be estimated by $\int_{\Omega}|u|_{\omega}^{2} \hat{\delta}^{-\eta} d V_{\omega}<+\infty$ if $\nu \leq n \eta-n+\ell$. But by assumption on $\eta$ we have $n \eta-n+\ell>0$; hence, we may deduce that for some small $\nu>0$ we have $u \in L_{n, n-1}^{2}\left(\Omega, \hat{\delta}^{1-\nu}\right)$.

But then for any $v \in \mathcal{C}_{0,1}^{\infty}(X)$ we have

$$
\begin{aligned}
\left|\int_{\Omega} u \wedge v\right|^{2} & \leq\left(\int_{\Omega}|u|_{\omega_{0}}^{2} \hat{\delta}^{1-\nu} d V_{\omega_{0}}\right) \cdot\left(\int_{\Omega}|v|_{\omega_{0}}^{2} \hat{\delta}^{-1+\nu} d V_{\omega_{0}}\right) \\
& \leq\|u\|_{\hat{\delta}^{1-\nu}}^{2} \cdot\left(\int_{\Omega} \hat{\delta}^{-1+\nu} d V_{\omega_{0}}\right) \sup _{\Omega}|v|_{\omega_{0}}^{2} .
\end{aligned}
$$

Since $\nu>0$, we have $\int_{\Omega} \hat{\delta}^{-1+\nu} d V_{\omega_{0}}<+\infty$. Therefore, $u$ defines a current $T \in \mathcal{D}_{0,1}^{\prime}(X)$.

It remains to see that $T=T_{u}$ satisfies $\bar{\partial} T=f$ in the sense of distributions on $X$. Let $\alpha \in \mathcal{C}_{0,0}^{\infty}(X)$. We must show that

$$
\int_{\Omega} u \wedge \bar{\partial} \alpha=\int_{\Omega} f \wedge \alpha
$$

Let $\chi \in \mathcal{C}^{\infty}(\mathbb{R}, \mathbb{R})$ be a function such that $\chi(t)=0$ for $t \leq 1 / 2$ and $\chi(t)=1$ for $t \geq 1$. Set $\chi_{j}=\chi(j \hat{\delta}) \in \mathcal{D}^{0,0}(\Omega)$. Then $\chi_{j} \alpha \in \mathcal{D}^{0,0}(\Omega)$, and since $\bar{\partial} u=f$ in $\Omega$, we therefore have

$$
\int_{\Omega} f \wedge \chi_{j} \alpha=\int_{\Omega} u \wedge \bar{\partial}\left(\chi_{j} \alpha\right)=\int_{\Omega} u \wedge\left(\alpha \bar{\partial} \chi_{j}+\chi_{j} \wedge \bar{\partial} \alpha\right) .
$$

As $f$ has $L^{2}$ coefficients on $\Omega$, the integral of $f \wedge \chi_{j} \alpha$ converges to the integral of $f \wedge \alpha$ as $j$ tends to infinity. The convergence of the integral of $u \wedge \chi_{j} \bar{\partial} \alpha$ to the integral of $u \wedge \bar{\partial} \alpha$ follows from $u \in L_{n, n-1}^{2}\left(\Omega, \hat{\delta}^{1-\nu}\right)$ (use the estimate (4.5)). 
The remaining term can be estimated as follows. Using the CauchySchwarz inequality we have

$$
\begin{aligned}
\left|\int_{\Omega} u \wedge \alpha \bar{\partial} \chi_{j}\right|^{2} & =\left|\int_{\left\{\frac{1}{2 j} \leq \hat{\delta} \leq \frac{1}{j}\right\}}\left\langle u \hat{\delta}^{-\eta / 2}, \overline{\star_{\omega} \alpha \bar{\partial} \chi_{j} \hat{\delta}^{\eta / 2}}\right\rangle_{\omega} d V_{\omega}\right|^{2} \\
& \leq \int_{\left\{\frac{1}{2 j} \leq \hat{\delta} \leq \frac{1}{j}\right\}}\left|u \hat{\delta}^{-\eta / 2}\right|_{\omega}^{2} d V_{\omega} \cdot \int_{\left\{\frac{1}{2 j} \leq \hat{\delta} \leq \frac{1}{j}\right\}}\left|\star_{\omega} \alpha \bar{\partial} \chi_{j} \hat{\delta}^{\eta / 2}\right|_{\omega}^{2} d V_{\omega} \\
& \leq \sup _{\Omega}|\alpha|^{2} \int_{\left\{\hat{\delta} \leq \frac{1}{j}\right\}}|u|_{\omega}^{2} \hat{\delta}^{-\eta} d V_{\omega} \cdot \int_{\Omega}\left|\bar{\partial} \chi_{j}\right|_{\omega}^{2} \hat{\delta}^{\eta} d V_{\omega}
\end{aligned}
$$

where $\star_{\omega}$ denotes the Hodge star operator with respect to $\omega$ in $\Omega$. Since $u \in L_{n, n-1}^{2}\left(\Omega, \hat{\delta}^{-\eta}, \omega\right)$, the integral $\int_{\{\hat{\delta} \leq 1 / j\}}|u|_{\omega}^{2} \hat{\delta}^{-\eta} d V_{\omega}$ converges to 0 when $j$ tends to infinity.

To estimate the second integral, we look at the behavior of its integrand $\left|\bar{\partial} \chi_{j}\right|_{\omega}^{2}$ near $\partial \Omega$. From $\bar{\partial} \chi_{j}=j \chi^{\prime} \bar{\partial} \hat{\delta}$,

$$
\begin{aligned}
\hat{\delta}(z)^{\eta-2}\left|\bar{\partial} \chi_{j}\right|_{\omega}^{2}(z) & \leq j^{2}\left\|\chi^{\prime}\right\|_{\mathcal{C}^{1}(\mathbb{R})}^{2}|\bar{\partial} \hat{\delta}|_{\hat{\delta}^{2-\eta} \omega}^{2}(z) \\
& =j^{2}\left\|\chi^{\prime}\right\|_{\mathcal{C}^{1}(\mathbb{R})}^{2} \max _{0 \neq v \in T_{z}^{1,0} X} \frac{|\partial \hat{\delta}(v)|^{2}}{\eta\left(\hat{\delta}(z) i \partial \bar{\partial}(-\hat{\delta})(v, v)+|\partial \hat{\delta}(v)|^{2}\right)} \\
& \rightarrow j^{2}\left\|\chi^{\prime}\right\|_{\mathcal{C}^{1}(\mathbb{R})}^{2} \frac{1}{\eta} \quad \text { as } z \rightarrow \partial \Omega
\end{aligned}
$$

Therefore, $\left|\bar{\partial} \chi_{j}\right|_{\omega}^{2} \lesssim j^{2} \hat{\delta}^{2-\eta}$ near $\partial \Omega$. Since the Levi form of $\partial \Omega$ has $\ell$ zero eigenvalues, we can estimate it with Lemma 3.1 as

$$
\begin{aligned}
\int_{\Omega}\left|\bar{\partial} \chi_{j}\right|_{\omega}^{2} \hat{\delta}^{\eta} d V_{\omega} & \lesssim \int_{\left\{\hat{\delta} \leq \frac{1}{j}\right\}} j^{2} \hat{\delta}^{2-\eta} \hat{\delta}^{\eta} \hat{\delta}^{n \eta-2-(n-\ell-1)} d V_{\omega_{0}} \\
& =\int_{\left\{\hat{\delta} \leq \frac{1}{j}\right\}} j^{2} \hat{\delta}^{1+n \eta-(n-\ell)} d V_{\omega_{0}} \\
& \lesssim j^{2-(2+n \eta-(n-\ell))} \\
& =j^{-n \eta+n-\ell} \rightarrow 0
\end{aligned}
$$

as $j \rightarrow \infty$ since $-n \eta+n-\ell<0$ by the assumption that $\eta>(n-\ell) / n$.

Therefore, $\int_{\Omega} u \wedge \alpha \bar{\partial} \chi_{j}$ converges to 0 when $j$ tends to infinity. Equation (4.6) follows. 


\section{§5. Proof of the Main Theorem}

The proof of the Main Theorem easily follows from Theorem 4.1 using a duality argument.

Proof of the Main Theorem. Assume by contradiction that the Levi form of the boundary $\partial \Omega$ has $\ell$ zero eigenvalues, and assume that $\eta(\Omega)>(n-\ell) / n$. Let $f \in \mathcal{D}^{n, n}(\Omega)$ be a smooth form of top degree with compact support in $\Omega$ satisfying $\int_{\Omega} f=1$. Applying Theorem 4.1, we can find a current $T \in \mathcal{D}_{0,1}^{\prime}(X)$ satisfying $\bar{\partial} T=f$ in the current sense. Let $\chi$ be a compactly supported smooth function on $X$ which is equal to one on $\bar{\Omega}$. But then

$$
1=\int_{\Omega} f=\langle f, \chi\rangle=\langle T, \bar{\partial} \chi\rangle=0 .
$$

This contradiction proves that $\eta(\Omega) \leq(n-\ell) / n$.

Acknowledgments. After this work was completed, the authors were kindly informed by Siqi Fu and Mei-Chi Shaw that they had already reached the same result for a weaker assumption (see [10]) with a different technique. We are grateful to them and to Takeo Ohsawa for communicating this information.

We also thank the referees for their careful reading and suggestions to improve the presentation of this article.

\section{REFERENCES}

[1] M. Adachi, On the ampleness of positive CR line bundles over Levi-flat manifolds, Publ. Res. Inst. Math. Sci. 50 (2014), 153-167. MR 3167582. DOI 10.4171/PRIMS/127.

[2] B. Berndtsson and P. Charpentier, A Sobolev mapping property of the Bergman kernel, Math. Z. 235 (2000), 1-10. MR 1785069. DOI 10.1007/s002090000099.

[3] J. Brinkschulte, The $\bar{\partial}$-problem with support conditions on some weakly pseudoconvex domains, Ark. Mat. 42 (2004), 259-282. MR 2101387. DOI 10.1007/BF02385479.

[4] J. Cao, M.-C. Shaw, and L. Wang, Estimates for the $\bar{\partial}-$ Neumann problem and nonexistence of $\mathcal{C}^{2}$ Levi-flat hypersurfaces in $\mathbb{C P}^{n}$, Math. Z. 248 (2004), 183221; Correction, Math. Z. 248 (2004), 223-225. MR 2092728; MR 2092729. DOI 10.1007/s00209-004-0661-0.

[5] D. Chakrabarti and M.-C. Shaw, $L^{2}$ Serre duality on domains in complex manifolds and applications, Trans. Amer. Math. Soc. 364, no. 7 (2012), 3529-3554. MR 2901223. DOI 10.1090/S0002-9947-2012-05511-5.

[6] J.-P. Demailly, Complex Analytic and Differential Geometry, preprint, https://www-fourier.ujf-grenoble.fr/ demailly/manuscripts/agbook.pdf (accessed September 16, 2015). 
[7] K. Diederich and J. E. Fornaess, Pseudoconvex domains: Bounded strictly plurisubharmonic exhaustion functions, Invent. Math. 39 (1977), 129-141. MR 0437806.

[8] - Pseudoconvex domains: An example with nontrivial Nebenhülle, Math. Ann. 225 (1977), 275-292. MR 0430315.

[9] H. Donnelly and C. Fefferman, $L^{2}$-cohomology and index theorem for the Bergman metric, Ann. of Math. (2) 118 (1983), 593-618. MR 0727705. DOI 10.2307/2006983.

[10] S. Fu and M.-C. Shaw, The Diederich-Fornaess exponent and non-existence of Stein domains with Levi-flat boundaries, published electronically 25 November 2014. DOI 10.1007/s12220-014-9546-6.

[11] P. S. Harrington and M.-C. Shaw, The strong Oka's lemma, bounded plurisubharmonic functions and the $\bar{\partial}-$ Neumann problem, Asian J. Math. 11 (2007), 127-139. MR 2304586. DOI 10.4310/AJM.2007.v11.n1.a12.

[12] T. Ohsawa and N. Sibony, Bounded p.s.h. functions and pseudoconvexity in Kähler manifolds, Nagoya Math. J. 149 (1998), 1-8. MR 1619572.

\author{
Masanori Adachi \\ Department of Mathematics \\ Faculty of Science and Technology \\ Tokyo University of Science \\ Tokyo \\ Japan \\ adachi_masanori@ma.noda.tus.ac.jp \\ Judith Brinkschulte \\ Universität Leipzig \\ Mathematisches Institut \\ D-04009 Leipzig \\ Germany \\ brinkschulte@math . uni-leipzig.de
}

